\title{
Magnetic flux distribution in a ferromagnetic material magnetized by U-shaped electromagnets of different geometric dimensions types
}

\author{
Anna Povolotskaya ${ }^{1, *}$, Eduard Gorkunov ${ }^{1}$, Pavel Nosov ${ }^{1}$, and Sergey Zadvorkin ${ }^{1}$ \\ ${ }^{1}$ Institute of Engineering Science, RAS (Ural Branch), Ekaterinburg, Russia
}

\begin{abstract}
A model experiment has been carried out on studying the type of changes in the magnetic induction in a homogeneous isotropic sample that is locally magnetized with attached U-type electromagnets of different geometrical dimensions. The study was aimed at finding out magnetic flux distribution at different locations within the sample and determining the effect that the geometry of attached electromagnets has on this distribution.
\end{abstract}

\section{Introduction}

Magnetic methods of non-destructive testing and evaluation are widely used for assessing the structural state, determining the strength characteristics, and analyzing the phase composition of products made of ferromagnetic materials [1-4]. In non-destructive testing of products under industrial conditions, magnetic characteristics are most often measured locally by using attached magnetic devices (AMD) that include magnetization facilities (coils, permanent magnets, electromagnets) and gaging elements (pickup coils, flux gates, Hall probes, magnetoresistors, etc.) [5]. The geometrical sizes of test products in most cases by far exceed the AMD sizes. Therefore, it is important that the magnetization facility should ensure magnetization or magnetization reversal of the evaluated area of the test product along a close-to-major hysteresis loop. In order to choose the optimum AMD configuration and adequately interpret the results of local magnetic measurements during in-production control of the structure and mechanical properties of metallurgical products (roll stock, forged pieces, rolls, etc.) without using costly "classical" testing methods, it is important to know magnetic flux distribution in near-surface and internal layers of test objects.

When evaluating surface-hardened products $[6,7]$, a more complicated problem arises. To assess the characteristics of the surface-hardened layer, it is necessary to concentrate magnetic flux in the hardened layer, whereas when evaluating properties of a viscous bulk of the product, one needs to ensure significantly deeper magnetic-flux penetration [8].

Thus, depending on the local magnetic evaluation task, the topography of magnetic-flux distribution in the product as well as the conditions of magnetization and magnetization

*Corresponding author:us@imach.uran.ru 
reversal in separate tubes of product's magnetic flux should be known [9].

Some experimental studies of magnetic-flux topography in ferromagnetic products that are locally magnetized by an attached electromagnet have been reported [10-14]. Theoretical calculations of the steadystate magnetic-flux distribution that are based on the system of Maxwell equations and the fundamental integral equation of magnetostatics have been performed only for some particular cases. Some works on the numerical modeling of flux and field distributions inside ferromagnets have been published recently [15-18]. In most experimental studies, methodological obstacles that are related to imperfection of measuring transducers impose certain restrictions on the shape of test products, and the shape, in its turn, affects the magnetic-flux distribution in these samples. For example, in [10] the width of the plate coincides with the width of electromagnet poles; this considerably reduces lateral magnetic-flux straying and increases magnetization depth. The same is confirmed by modeling results in [15]. Miniature high-sensitivity measuring transducers are currently used to take measurements of magnetic-flux parameters without the above restrictions on the sample shape.

The aim of this work is to study experimentally the type of changes in magnetic induction in a homogeneous isotropic sample with allowance for lateral straying when the sample is locally magnetized by attached U-type electromagnets of different sizes in order to examine the spatial magnetic-flux distribution in the sample and to determine the effect that the geometry of attached electromagnets has on this distribution.

\section{Experimental procedure and material}

A compound model sample of $40 \mathrm{Kh}$ chromium steel, which is extensively used in machine building, was prepared for the experiments. The sample consisted of two parts A and B with dimensions $100 \times 70 \times 90$ and $85 \times 70 \times 90 \mathrm{~mm}$, respectively. A schematic drawing of the sample is presented in Fig. 1.

A $10 \times 10-\mathrm{mm}$ groove was milled on the inner surface of part $A$ that is in contact with part B. A $10 \times 10 \times 10-\mathrm{mm}$ measuring insert made of steel of the same grade could be displaced along the entire groove length. Three Hall probes were attached to each of the three mutually orthogonal facets of the measuring insert. The insert is schematically shown in Fig. $1 b$.

Auxiliary 40Kh-steel $10 \times 10$-mm inserts with lengths of 2, 5, 10, 20, 40, and $50 \mathrm{~mm}$ were manufactured to ensure the homogeneity of the magnetic medium that was tested. These inserts were used to fill the entire length of the groove. During experiments, parts A and $\mathrm{B}$ were fayed together, with the measuring insert being fixed in the groove. To avoid distortions of magnetic flux in the model sample, all the above parts were subjected to identical thermal treatment (quenching at a temperature of $860^{\circ} \mathrm{C}$ followed by tempering at a temperature of $300{ }^{\circ} \mathrm{C}$ in a vacuum furnace) so as to ensure identical magnetic and mechanical properties.

The geometrical dimensions and the number of turns of electromagnets that were used to magnetize the sample are listed in Table 1.

Table 1. Geometrical dimensions of used U-type electromagnets.

\begin{tabular}{|c|c|c|c|c|}
\hline $\begin{array}{c}\text { AMD } \\
\text { no. }\end{array}$ & $\begin{array}{c}\text { Pole thickness } a, \\
\mathrm{~mm}\end{array}$ & $\begin{array}{c}\text { Pole width } b, \\
\mathrm{~mm}\end{array}$ & $\begin{array}{c}\text { Distance between pole centers } \\
d, \mathrm{~mm}\end{array}$ & $\sqrt{S_{\mathrm{e}}, \mathrm{mm}}$ \\
\hline 1 & 11.5 & 28 & 45.5 & 17.9 \\
\hline 2 & 5.5 & 15 & 18.5 & 9.08 \\
\hline 3 & 10 & 25 & 23 & 15.8 \\
\hline
\end{tabular}


The electromagnet current varied within a range of -2.6 to $+2.6 \mathrm{~A}$ using a regulated power supply unit. The origin of a Cartesian coordinate system was put at the center of electromagnet's neutral cross section, as show in Figs. $2 a$ and $2 b$. The Hall probes that registered induction along $x$-, $y$-, and $z$-axes are denoted as $P_{x}, P_{y}$, and $P_{z}$, accordingly. At the beginning of measurements, the electromagnet was placed on the preliminarily demagnetized sample so that the Hall probe $P_{x}$ be at the center of the neutral cross section of the electromagnet at a depth of $5 \mathrm{~mm}$ [at a point with coordinates $(0 ; 0 ; 5)]$.
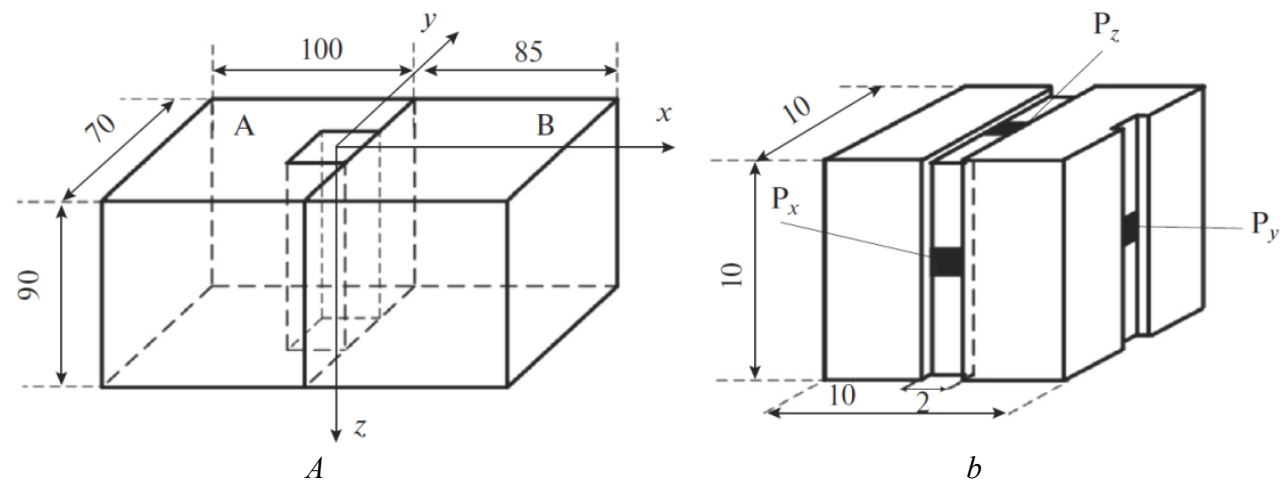

Fig. 1. Schematic drawings of ( $a$ ) sample and $(b)$ measuring insert.

Measurements with electromagnets were carried out in the following manner. The measuring insert was installed so that its upper facet should coincide with the surface of the model sample (see Fig. 2a). Given such an arrangement, the Hall probes had the following coordinates: $P_{x}(x=0, y=0, z=5) ; P_{y}(x=-5, y=5, z=5)$; and $P_{z}(x=-5, y=0, z=10)$. A cycle of measurement of magnetic properties was executed in this initial position (see Fig. $2 b$ ). The entire system was demagnetized in all cases prior to the measurements, with the complete demagnetization performed after each measurement cycle.
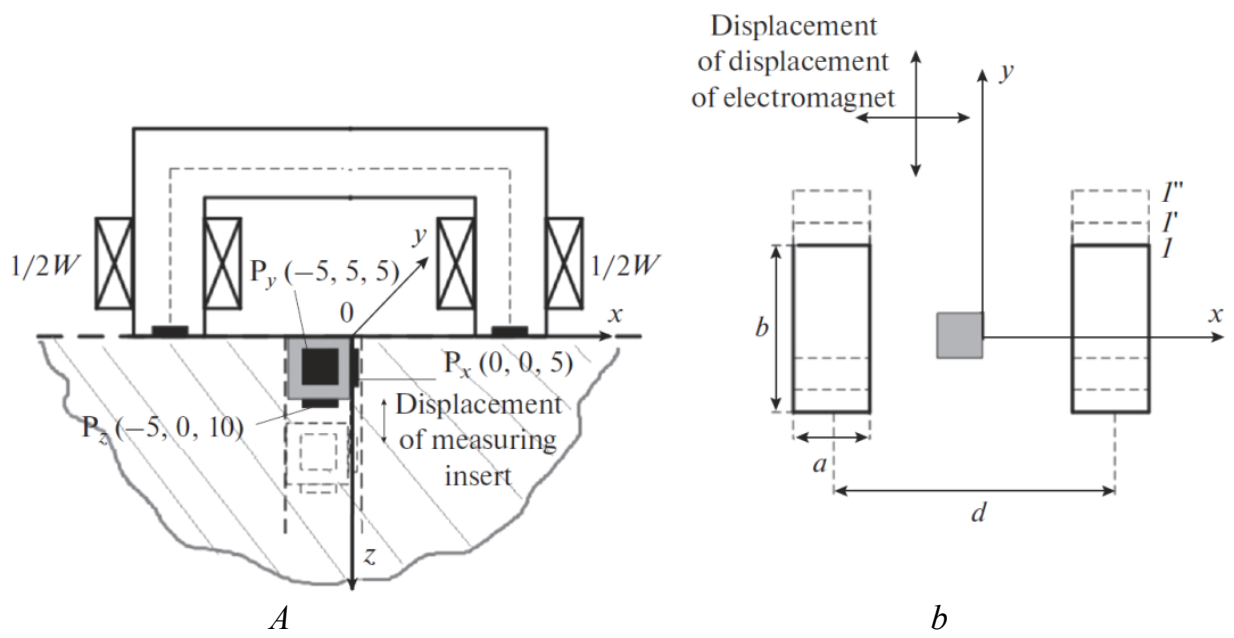

Fig. 2. Disposition of electromagnet on the sample and orientation of Hall probes $(a)$; electromagnet displacement (view from above) (b). 
In order to study lateral straying, the attached electromagnet was sequentially displaced along the $y$-axis in 5-mm steps into positions 1', 1" and so on (see Fig. 2b), with the entire measurement cycle of hysteresis loops by the Hall probes repeated every time. Then, the electromagnet was returned to the original position, and similar measurements were taken, with the electromagnet displaced in 5-mm steps along the $x$-axis. After measurements for different prescribed values of $x$ had been taken, the electromagnet returned to the initial position.

The next series of measurements were taken after sequential 5-mm displacements of the measuring insert, with the free space filled every time with the auxiliary inserts.

\section{Results and discussion}

Figure 3 shows how the value of the $x$-component of magnetic induction $B_{x}$ as measured with AMD 1 and AMD 2 for the maximum applied field depends on the distance $x$ to the plane of AMD's neutral cross section for different positions along the $y$-axis at a depth of $z$ $=5 \mathrm{~mm}$ from the sample surface.

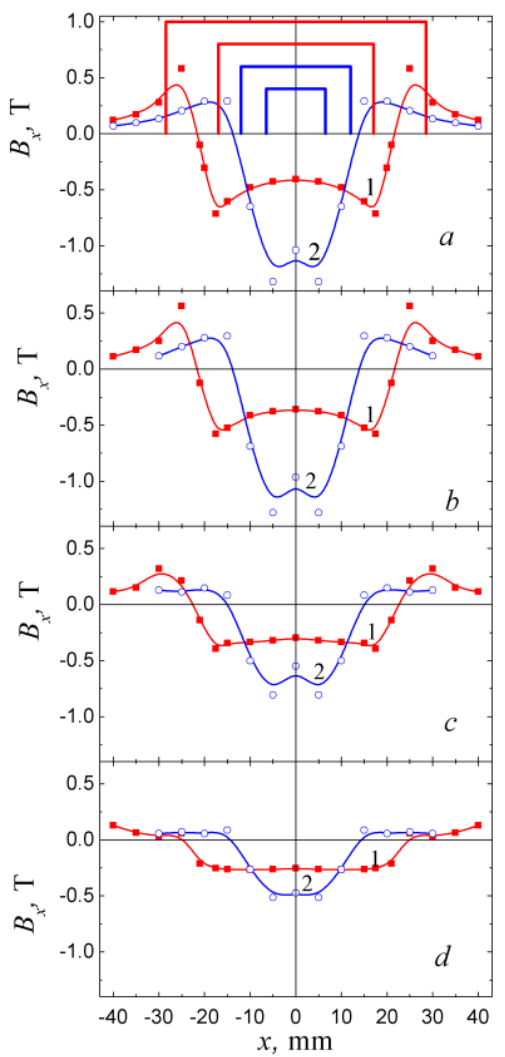

Fig. 3. Schematic arrangement of AMD 1 and AMD 2 on the sample and values of magnetic induction components $B_{x}$ at different distances from the center of AMD neutral cross section at depth $z=5 \mathrm{~mm}$ from the sample surface at (a) $y=0$; (b) 5; (c) 15; and (d) $20 \mathrm{~mm}$. Curves 1 and 2 are obtained with AMD 1 and AMD 2, respectively.

For the sake of clearness, Fig. $3 a$ schematically presents attached magnetic devices. It can be seen that the $x$-component of magnetic induction $B_{x}$-both for AMD 1 and for AMD 
2 - increases when approaching the electromagnet pole starting at half the interpolar gap, attains its maximum approximately at the border of the inner pole edge, and then decreases to zero. When the electromagnet moves still further away from the measuring insert, $B_{x}$ changes sign, reaches its maximum near the outer pole edge, and then rapidly drops down to zero. When the AMD is displaced from the measuring insert along the $y$-axis, the $x$ component of magnetic induction $B_{x}$ decreases, the maxima near the borders of the poles become less pronounced, and at $y=20 \mathrm{~mm}$ the $x$-component of the magnetic-flux density only slightly changes in the interpolar zone.

The $x$-component of the magnetic induction $B_{x}$ changes in such a way only at a relatively small depth of $z=5 \mathrm{~mm}$. At $z=10 \mathrm{~mm}$ (Fig. $4 a$ ), the maxima near the borders become less pronounced (for any displacement along the $y$-axis), while the sign-reversal position of $B_{x}$ is displaced farther from the center. At $z=20 \mathrm{~mm}$ (Fig. $4 b$ ), the $x$-component of magnetic induction $B_{x}$ does not change sign for any of the values of the $x$-coordinate that are indicated in the figures.

Figure 5 shows dependences of the $z$-component of the induction $B_{z}$ for the maximum applied field for different positions along the $y$-axis $(y=0,5,10,15,20 \mathrm{~mm})$ at the depth of $z=5 \mathrm{~mm}$. For clearness, the figures schematically indicate AMDs. The magnetic-induction component $B_{\mathrm{z}}$ at the center of the interpolar gap equals zero for any displacement along the $y$-axis, increases when approaching the pole, reaches its maximum value beneath the pole center, and decreases to zero when moving further away from the measuring insert. For deeper locations of the measuring insert, the values of $B_{z}$ are smaller, but the overall type of the dependences is retained.

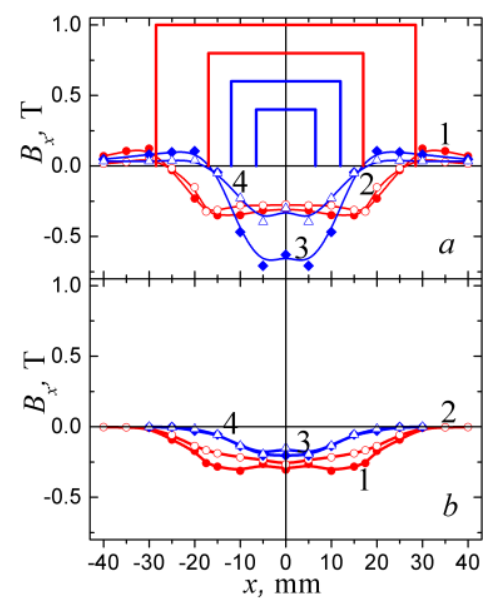

Fig. 4. Schematic arrangement of AMD 1 and AMD 2 on the sample and values of magneticinduction components $B_{x}$ for different positions of the plane $x, y$ at depths $z=(a) 10$ and $(b) 20 \mathrm{~mm}$ from the sample surface for $y=5 \mathrm{~mm}-$ curves $1(\bullet)$, and $3(\bullet)$, for $y=20 \mathrm{~mm}-$ curves 2 (o) and 4 $(\Delta)$. Curves $1(\bullet), 2(\mathrm{o})$ and curves $3(\bullet), 4(\Delta)$ were obtained from measurements with AMD 1 and AMD 2, respectively.

For the maximum applied field, the magnetic-induction component $B_{y}$ (Fig. 6) depends on the displacement along the $y$-axis in the following way. At $y=0 \mathrm{~mm}, B_{y}$ is equal to zero everywhere; then it grows when approaching the pole, reaches its maximum value near the border of the inner pole edge, and decreases to zero when the AMD moves further away from the measuring insert.

Displacement along the $y$-axis alters the type of the dependence of $B_{y}$ in the following manner. The maximum position is shifted closer to the pole center, whereas the very 
maximum value of $B_{y}$ first increases, reaches its maximum at $y=10-15 \mathrm{~mm}$, and then reduces (Fig. 7). The lack of monotony and the presence of a maximum on the $B_{y}(y)$ curve can be explained by the fact that in this case two "concurrent" processes occur. On the one hand, magnetic-flux density should in toto decrease to zero when the AMD is displaced along the $y$-axis, but, on the other hand, the ratio $B_{y} /|B|$ monotonically increases from zero and does not exceed unity, given such a displacement.

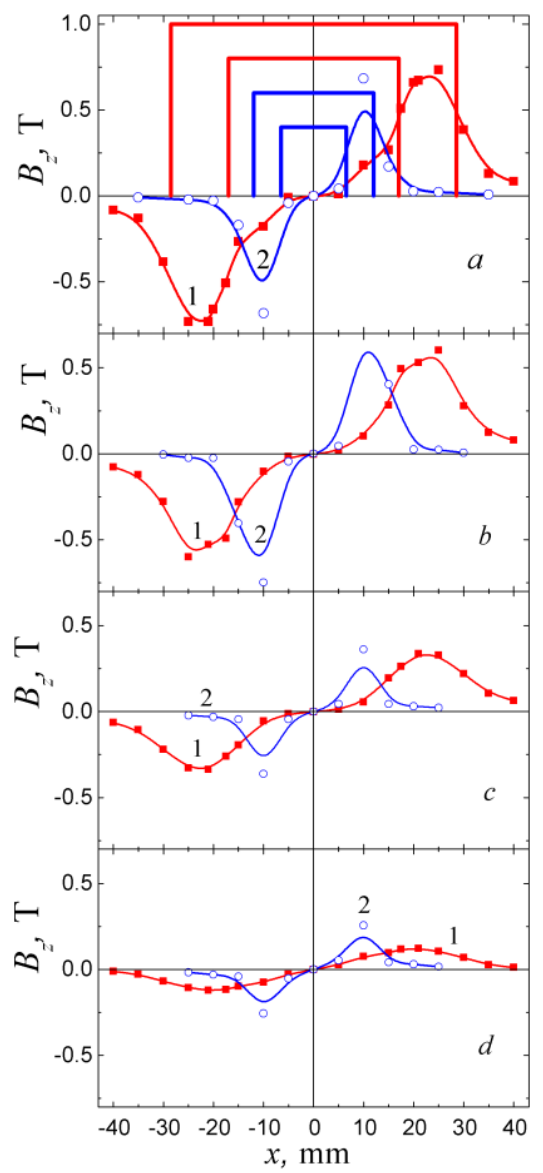

Fig. 5. Schematic arrangement of AMD 1 and AMD 2 on the sample and values of magneticinduction components $B_{z}$ at different distances from the center of AMD neutral cross section at depth $z=5 \mathrm{~mm}$ from the sample surface for $(a) y=0$; (b) 5; (c) 15; and (d) $20 \mathrm{~mm}$. Curves 1 and 2 were obtained with AMD 1 and AMD 2, respectively.

Figure 8 presents dependences of the modulus of the total magnetic-induction vector $|B|$ for the maximum applied field for different positions along the $y$-axis $(y=0,5,10,15,20$ $\mathrm{mm}$ ) at depths of $z=5,10,20 \mathrm{~mm}$, as measured with AMD 1. It can be seen that the modulus of the total magnetic-induction vector $|B|$ increases when approaching the pole and reaches its maximum value at the inner pole edge $(x=17 \mathrm{~mm})$. At the pole center, the value of $|B|$ decreases and then reaches its second maximum approximately $2-3 \mathrm{~mm}$ away from the outer pole edge $(x=28 \mathrm{~mm})$. As the AMD moves further away from the measuring insert, the modulus of the total magnetic-induction vector $|B|$ reduces and tends to zero. Such a type of changes in $|B|$ takes place only at a relatively small depth of $z=5 \mathrm{~mm}$. At $z=$ 
10 and $20 \mathrm{~mm}$, the first maximum (near the inner pole edge) stops being very pronounced, while the second maximum (near the pouter pole edge) disappears. Displacement of the AMD along the $y$-axis also diminishes the maxima of $|B|$ near the edges of the poles.

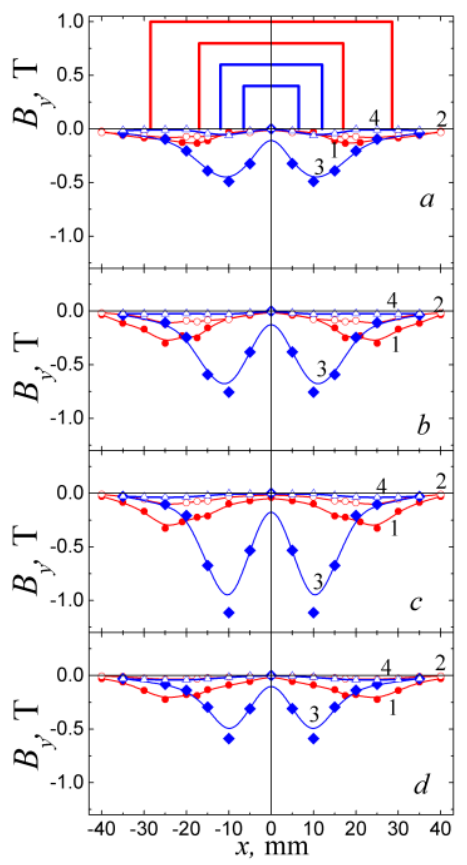

Fig. 6. Schematic arrangement of AMD 1 and AMD 2 on the sample and values of magneticinduction components $B_{y}$ for different positions of the plane $x, y$ at $y=(a) 5 ;(b) 10 ;(c) 15$; and (d) 20 $\mathrm{mm}$ at depths $z=5 \mathrm{~mm}$ [curves $1(\bullet)$ and $3(\bullet)$ ] and $z=20 \mathrm{~mm}$ [curves $2(\mathrm{o})$ and $4(\Delta)$ ] from the sample surface. Curves $1(\bullet), 2$ (o) and curves $3(\bullet), 4(\Delta)$ were obtained from measurements with AMD 1 and AMD 2, respectively.

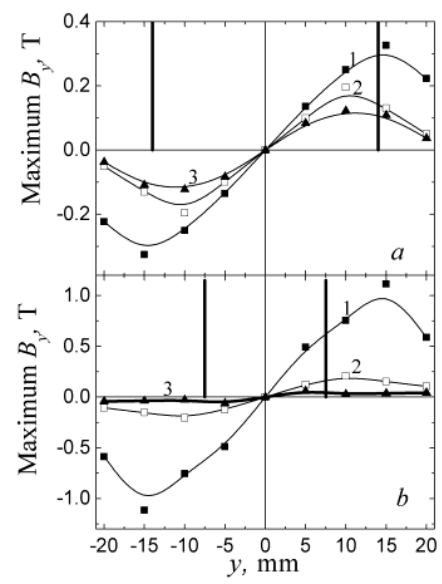

Fig. 7. Maximum values of magnetic-induction component $B_{y}$ depending on the displacement along the $y$-axis: $(a)$ measurements with AMD 1, (b) measurements with AMD 2. Curves 1, 2, and 3 correspond to $z=5 ; 10$; and $20 \mathrm{~mm}$, respectively. 
The fact that the maxima are located near pole edges can be explained as follows. The main contribution to the value of the modulus of the total magnetic-induction vector is rendered by the $B_{x}$ and $B_{z}$ components (the $B_{y}$ component contributes much less). However, the magnetic-induction component $B_{x}$ turns to zero approximately beneath the center of a pole of the attached magnetic device (see Figs. 3 and 4), thereby reducing the modulus $|B|$. Therefore, a small decrease in the modulus of magnetic-induction vector is observed beneath the pole center as compared with the pole edges. At $z=20 \mathrm{~mm}$, the component $B_{x}$ no longer demonstrates sign reversal beneath the pole, which is manifested in the lack of double maxima of $|B|$. It should be noted that the sign reversal of $B_{x}$ beneath the pole center is accounted for by the presence of stray fluxes in the transducer-sample circuit.

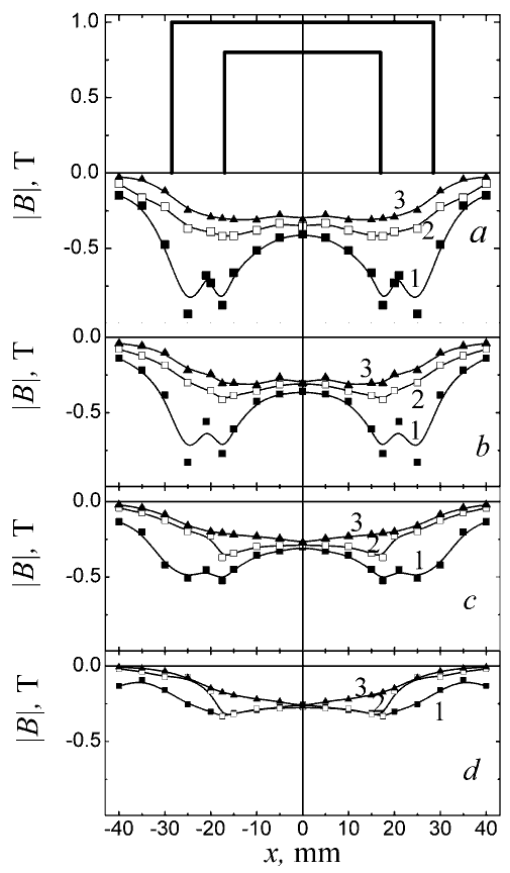

Fig. 8. Magnetic-induction modulus $|B|$ at different points of the sample for depths $z=5 \mathrm{~mm}$ (curves 1), $10 \mathrm{~mm}$ (curves 2), and $20 \mathrm{~mm}$ (curves 3) at $y=(a) 0$; (b) 5; (c) 10; and (d) $20 \mathrm{~mm}$. Measurements are taken with AMD 1.

When magnetizing products, it is important to know the depth to which they are magnetized. Figure 9 presents dependences of the modulus of the total magnetic-induction vector $|B|$ at the center of the neutral cross section $(x=0, y=0)$ on the distance $z$ to the sample surface (Fig. $9 a$ ) and on $z / \sqrt{ } S_{\mathrm{e}}$ (Fig. $9 b$ ). The dependences were measured when the sample was magnetized by three electromagnets with different geometrical dimensions, where $S_{\mathrm{e}}$ is the cross section area of the relevant AMD. The values of currents in measurements with the electromagnets of AMD 2 and AMD 3 were set so as to ensure a field of $4.2 \mathrm{kA} / \mathrm{m}$ beneath their poles, a field which is created beneath the pole in case of magnetization with AMD 1 operating at the maximum current. It can be seen from Fig. $9 a$ that the value of the total magnetic-induction vector $|B|$ monotonically decreases with the depth $z$, with its value dependent, to a considerable degree, on the geometrical dimensions of the attached magnetic device (the cross section area of the poles of the electromagnet and the interpolar distance). It should be noted that at small depths, in near-surface layers of the neutral section, the smaller the gap, the greater the values of $|B|$; whereas the greater the 
cross section area of the AMD poles, the larger the magnetization depth. The latter statement is illustrated more visually in Fig. $9 b$. At small depths of less than approximately $1.3 \sqrt{S_{\mathrm{e}}} \mathrm{mm}$, the value of $|B|$ depends, to a considerable degree, on the distance $d$ between the centers of electromagnet poles, while at larger depths it only depends on the cross section area of AMD pole. Reducing the pole section area, in particular, its width, leads to increasing lateral straying and, accordingly, decreasing the magnetization depth and flux density in the test object.
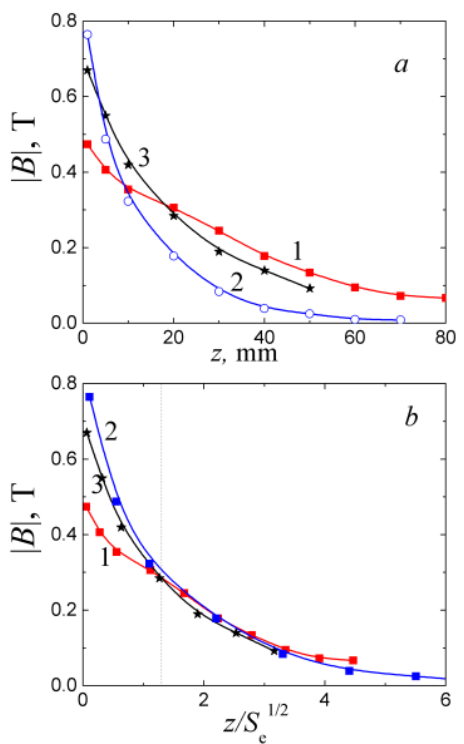

Fig. 9. Dependence of the modulus of total magnetic-induction vector $|B|$ at the center of the neutral cross section $(a)$ on the distance $z$ to the sample surface and $(b)$ on the value of $z / \sqrt{ } S_{\mathrm{e}}$. Curves 1,2 , and 3 were obtained with AMD 1, AMD 2, and AMD 3, respectively.

Kostin et al. [17] performed numerical modeling and obtained quantitative estimates of the lateral straying and percentage of magnetic flux that goes beyond the profile of electromagnet poles from the overall magnetic flux in the magnetic circuit. They demonstrated that, first of all, the greatest lateral straying of magnetic flux is observed in the neutral cross section, and, secondly, the lateral straying depends, to a considerable degree, on both the ratio of the width of the magnetized plate to the pole width and the interpolar distance, provided the cross sections of the poles are equal. Based on the experimental data for all the AMDs, the fraction of magnetic flux that leaves the boundaries of the poles to the overall magnetic flux that passes through the neutral cross section was calculated. The magnetic flux $B S$ was calculated by dividing the neutral cross section into square $5 \times 5$-mm cells and assuming the induction to be the same within any cell (Table 2 ).

Table 2. Fraction of the lateral straying of magnetic flux in the neutral cross section of the plate when it is magnetized by AMDs with different geometrical dimensions.

\begin{tabular}{|c|c|c|c|}
\hline AMD no. & $\begin{array}{c}\text { Ratio of plate } \\
\text { width to AMD pole width }\end{array}$ & $\begin{array}{c}\text { Ratio of distance between } \\
\text { AMD pole } \\
\text { centers to pole width }\end{array}$ & Lateral straying, \% \\
\hline 1 & 2.5 & 1.6 & 57 \\
\hline 2 & 4.7 & 1.2 & 65 \\
\hline 3 & 2.8 & 0.92 & 60 \\
\hline
\end{tabular}


As compared to AMD 3, AMD 1 has almost a twice larger distance between the pole centers, with the pole width of AMD 1 exceeding that of AMD 3 by only 1.12 times. It can be seen from the results provided in the table that reduction in lateral straying of magnetic flux in the neutral cross section and, hence, growth of the magnetization depth are affected to a much greater degree by reduction in the ratio of the width of the magnetized plate to the AMD pole width than by decrease in the ratio of the interpolar gap to the pole width.

\section{Conclusion}

A technique has been developed for determining the topography of magnetic induction in a ferromagnet that is magnetized by attached magnetic device. The distributions of all the magnetic-induction components over the volume of a bulky homogeneous isotropic sample of steel $40 \mathrm{Kh}$ that is locally magnetized by attached U-type electromagnets with different geometries.

It has been established that if the width of the magnetized plate is greater than the electromagnet-pole width, the distribution of magnetic flux in the plate at a distance to the plate surface of less than $1.3 \sqrt{S_{\mathrm{e}}}$ is affected by both the cross section area of AMD poles and the distance between the centers of the poles of the electromagnet.

It has been demonstrated experimentally that the main factor that significantly affects the growth of the magnetization depth is reduction of the ratio of the magnetized plate width to the AMD pole width due to reduction of the fraction of strayed magnetic flux that occurs in this case.

The above results can prove useful in magnetic nondestructive testing when choosing the most expedient disposition of measuring transducers within the space between electromagnet poles, magnetic structural analysis when adjusting the depth of magnetization of a test product as well as verifying the adequacy of numerical modeling of processes of magnetization and magnetization reversal of products made of steel $40 \mathrm{Kh}$.

The study was partially supported by UB RAS project No 15-10-1-22.

\section{References}

1. M. N. Mikheev, E. S. Gorkunov, Magnetic Methods of Structural Analysis and Nondestructive Testing (Nauka, Moscow, 1993)

2. V. V. Klyuev, V. N. Filinov, V. I. Matveev, Nondestructive Testing. A Handbook. In 8 Vols. Magnetic Methods of Testing, Vol. 6, Book 1 (Mashinostroenie, Moscow, 2006)

3. E. S. Gorkunov, Diagn. Resour. Mech. Mater. Struct. 1, 6-40 (2015)

4. E. S. Gorkunov, Diagn. Resour. Mech. Mater. Struct. 3, 6-50 (2015)

5. E. S. Gorkunov, V. A. Zakharov, Russ. J. Nondestr. Test. 31, 8, 625-641 (1995)

6. E. S. Gorkunov, V. A. Zakharov, Soviet J. Nondestr. Test. -USSR, 27, 1, 1-18 (1991)

7. L. Jian, K. T. Chau, Progress in Electromagnetics Research 92, 1-16, (2009)

8. V. E. Shcherbinin, E. S. Gorkunov, Magnetic Testing of Quality of Metals (Ural Branch, Russ. Acad. Sci., Ekaterinburg, 1996)

9. V. A. Zakharov, Magnetostatics of Systems with Ferromagnets (Ural Sci. Center, USSR Acad. Sci., Sverdlovsk, 1986)

10. M. N. Mikheev, Izv. Akad. Nauk SSSR, 3, 68-77 (1948)

11. G. V. Bida, Russ. J. Nondestr. Test. 35, 9, 702-711 (1999) 
12. G. V. Bida, E. Y. Sazhina, Russ. J. Nondestr. Test. 32, 5, 411-417 (1996)

13. L. A. Fridman, V. P. Tabachnik, G. S. Chernova, Soviet J. Nondestr. Test.-USSR, 13 , 4, 449-455 (1977)

14. M. N. Mikheev, Tr. Inst. Phys. Metals, 21 (1959)

15. O. N. Vasilenko, V. N. Kostin, Russ. J. Nondestr. Test. 49 (9), 510-518 (2013)

16. V. R. Skal'skii, B. P. Klim, E. P. Pochapskii, Russ. J. Nondestr. Test. 48 , 1, 23-34 (2012)

17. V. N. Kostin, O. N. Lukinykh, Y. G. Smorodinskii, K. V. Kostin, Russ. J. Nondestr. Test. 46, 6, 403-410 (2010)

18. V. N. Kostin, O. N. Vasilenko, A. M. Porseva, A. A. Kabakova, A. I. Melchakova, Diagn. Resour. Mech. Mater. Struct. 5, 8-15 (2015) 